\title{
Effect of Channel Variability on Pilot Design for Joint Communications and Positioning in OFDM
}

\author{
Arash Shahmansoori, Rafael Montalban and Gonzalo Seco-Granados \\ Universitat Autònoma de Barcelona \\ Bellaterra, Barcelona 08193, Spain \\ \{arash.shahmansoori, rafael.montalban, gonzalo.seco\}@uab.cat
}

\begin{abstract}
The accuracy of the estimation of time-delay and channel coefficients in Orthogonal frequency division multiplexing (OFDM) communication systems can be improved by reducing the variability of channel coefficients, i.e. reducing channel covariance and increasing channel mean for a given power. First, we prove that the effect of channel variability between different OFDM symbols cannot be directly captured by extending expected Crámer Rao bound (ECRB) for one OFDM symbol to $M$ OFDM symbols. Then, the effect of channel variations between different OFDM symbols is modeled as the variations of channel covariance and channel mean for one OFDM symbol and a given channel power. A pilot design approach based on per-symbol signal to noise and interference ratio (SINR) for a given time-delay estimation accuracy is investigated. The results show that reducing channel covariance and improving channel mean for a given channel power leads to more accurate estimation of time-delay and channel coeficients. Furthermore, one can save the total power for a given estimation accuracy and channel capacity.
\end{abstract}

Index Terms-OFDM, ECRB, joint pilot design, SINR, channel variability.

\section{INTRODUCTION}

Location awareness is one of the fundamental characteristics of cognitive radio (CR). Applications of location awareness can require different level of positioning accuracy. For instance, indoor positioning usually requires higher positioning accuracy. Also, one of the crucial aspects for developing a cooperative positioning system is to establish an accurate positioning method. On the other hand, one needs to keep the communications rate above a certain level at the same time. Therefore, designing a sequence that can satisfy both requirements simultaneously based on the system specifications for time-delay estimation (This can be converted to the distance measurements) accuracy and channel capacity is of interest.

Pilot symbols are widely used for estimation and synchronization in communications and navigation systems. For instance, pilot design in the estimation of time-delay can be used for ranging in global navigation satellite systems (GNSS). Since the choice of pilot design significantly affects estimation performance, it is of critical importance to find the optimal pilots that can improve the estimation accuracy.

This work was financially supported by EU FP7 Marie Curie Initial Training Network MULTI-POS (Multi-technology Positioning Professionals) under grant nr. 316528 .

978-1-4799-5863-4/14/\$31.00 (C)2014 IEEE
To date, different approaches have been adopted to design pilot symbols that improve the performance of channel estimators [1]-[3]. The results show that equi-spaced, equipowered pilots are optimal in terms of minimum mean square error (MMSE). Pilot designs based on carrier frequency offset (CFO) estimation [4], or joint channel and CFO estimation [5] are considered by others. However, pilot design based on joint time-delay and channel estimation has received little attention. In [6], a pilot design for joint channel and time-delay based on hybrid CRB (CRB) is presented. However, the bound is not a tight bound comparing to the other bounds, e.g. ECRB. A Pilot design based on joint CRB of channel and time-delay is proposed in [7]. However, since CRBs in [7] are functions of specific channel realizations, the resulting pilots are not guaranteed to be optimal for all instances of random channels. The problem has been solved by designing based on averaging the CRB over a set of channel realizations known as ECRB. Note that in [7], [8], it is assumed that the design is based on one OFDM symbol.

In this paper, we use ECRB for joint time-delay and channel coefficients [8] to avoid the design for only a specific type of channel and rather dealing with channel statistics. On the one hand, the effect of channel variations between different OFDM symbols is not captured by extending the model for one OFDM symbol to several symbols. On the other hand, channel variations between different OFDM symbols can be modeled as variations of channel covariance and channel mean for a given power. Sequential methods for updating MMSE for different symbols (e.g., Kalman filters) provides insight to our approach of updating channel covariance and channel mean between different OFDM symbols. However, applying sequential methods of updating MMSE of timedelay and channel coefficients requires the estimation of timedelay as a non-linear parameter resulting the use of extended Kalman filters [9] which is beyond the scope of this paper. Consequently, we use an alternative approach by changing channel covariance and mean for a certain channel power to model variations between different OFDM symbols. A pilot design approach for optimization based on per-symbol signal to interference and noise ratio (SINR) for a given time-delay estimation accuracy is investigated. Results show a significant improvement in terms of estimation accuracy and saving the total power by reducing channel variability. 


\section{Signal Model And ECRB}

In this section the signal model and ECRB of time-delay $\mathrm{ECRB}_{\tau_{d}}$ and the trace of ECRB of channel coefficients $\operatorname{tr}\left\{\mathrm{ECRB}_{h}\right\}$ for one OFDM symbol and $M$ OFDM symbols are briefly explained.

\section{A. Signal Model}

We use the following OFDM channel model

$$
\boldsymbol{Y}=\boldsymbol{\Omega}\left(\tau_{d}\right) \boldsymbol{h}+\boldsymbol{W},
$$

where

$$
\boldsymbol{\Omega}\left(\tau_{d}\right)=\boldsymbol{X} \boldsymbol{\Gamma}\left(\tau_{d}\right) \boldsymbol{F}_{L},
$$

and $\boldsymbol{X}$ represents an $N \times N$ diagonal matrix of the input with the $k$ th diagonal element $X[k]$ representing the input at the $k$ th subcarrier for $k=-\frac{N}{2}+1, \ldots, \frac{N}{2}$ for even values of $N$ and $k=-\frac{N-1}{2}, \ldots, \frac{N-1}{2}$ for odd values of $N$, $\boldsymbol{\Gamma}\left(\tau_{d}\right)$ is an $N \times N$ diagonal matrix with the $k$ th diagonal element $\exp \left(-j 2 \frac{\pi}{T_{s}} k \tau_{d}\right)$ where $\tau_{d}$ is the time-delay, $T_{s}$ is the OFDM symbol length, $\boldsymbol{F}_{L}$ contains the first $L$ columns of a Discrete Fourier Transform (DFT) matrix centered around zero, $\boldsymbol{h}$ is an $L \times 1$ column vector representing channel coefficients, $\boldsymbol{W}$ is zero-mean complex Gaussian noise distributed as $\mathcal{C N}\left(\mathbf{0}, \sigma_{w}^{2} \boldsymbol{I}\right)$, and finally $\boldsymbol{Y}$ is an $N \times 1$ vector representing the output signal.

\section{B. ECRB for One OFDM Symbol}

Using the Fisher Information Matrix (FIM) for the joint estimation of time-delay $\tau_{d}$ and channel coefficients $\boldsymbol{h}$ [7], one can obtain the CRB. However, CRB depends on the channel coefficients $\boldsymbol{h}$. To find a bound that does not depend on the channel coefficients $\boldsymbol{h}$ but channel statistics, i.e. channel mean $\boldsymbol{\mu}_{h}$ and covariance $\boldsymbol{\Sigma}_{h}$, one can take the expectation of CRB with respect to channel coefficients $\boldsymbol{h}$ and use the Laplace approximation, i.e. $\mathbb{E}\left[\frac{X}{Y}\right] \approx \frac{\mathbb{E}[X]}{\mathbb{E}[Y]}$, to obtain the approximated ECRB as [8]

$$
\left[\begin{array}{c}
\mathrm{ECRB}_{\tau_{d}} \\
\operatorname{tr}\left\{\mathrm{ECRB}_{h}\right\}
\end{array}\right] \approx \frac{\sigma_{w}^{2}}{2}\left[\begin{array}{c}
J_{\tau_{d}}^{-1} \\
J_{h}
\end{array}\right]
$$

where

$$
J_{\tau_{d}}=\operatorname{tr}\left\{\left(M_{1}-M_{2}\right) \boldsymbol{\Sigma}_{h}\right\}+\boldsymbol{\mu}_{h}^{H}\left(M_{1}-M_{2}\right) \boldsymbol{\mu}_{h},
$$

and

$$
J_{h}=2 \operatorname{tr}\left\{\boldsymbol{Q}^{-1}\right\}+J_{\tau_{d}}^{-1}\left(\operatorname{tr}\left\{\boldsymbol{M}_{q} \boldsymbol{\Sigma}_{h}\right\}+\boldsymbol{\mu}_{h}^{H} \boldsymbol{M}_{q} \boldsymbol{\mu}_{h}\right),
$$

with $\boldsymbol{M}_{1}, \boldsymbol{M}_{2}$, and $\boldsymbol{M}_{q}$ defined as $\boldsymbol{M}_{1}=\boldsymbol{F}_{L}^{H} \boldsymbol{D P} \boldsymbol{D} \boldsymbol{F}_{L}, \boldsymbol{M}_{2}=\boldsymbol{F}_{L}^{H} \boldsymbol{P} \boldsymbol{D} \boldsymbol{F}_{L} \boldsymbol{Q}^{-1} \boldsymbol{F}_{L}^{H} \boldsymbol{P} \boldsymbol{D} \boldsymbol{F}_{L}$ and $\boldsymbol{M}_{q}=\boldsymbol{F}_{L}^{H} \boldsymbol{P} \boldsymbol{D} \boldsymbol{F}_{L} \boldsymbol{Q}^{-1} \boldsymbol{Q}^{-1} \boldsymbol{F}_{L}^{H} \boldsymbol{P} \boldsymbol{D} \boldsymbol{F}_{L}$ respectively. Also, $\boldsymbol{Q}=\boldsymbol{F}_{L}^{H} \boldsymbol{P} \boldsymbol{F}_{L}, \boldsymbol{P}=\boldsymbol{X}^{H} \boldsymbol{X}$ is an $N \times N$ diagonal matrix representing pilot powers, $\boldsymbol{D}=\frac{2 \pi}{T_{s}} \times$ $\operatorname{diag}\left\{-\frac{N}{2}+1, \ldots, \frac{N}{2}\right\}$ for even values of $N$ and $\boldsymbol{D}=$ $\frac{2 \pi}{T_{s}} \times \operatorname{diag}\left\{-\frac{N-1}{2}, \ldots, \frac{N-1}{2}\right\}$ for odd values of $N, \boldsymbol{\mu}_{h}=\mathbb{E}[\boldsymbol{h}]$ is the channel mean, and $\boldsymbol{\Sigma}_{h}=\mathbb{E}\left[\left(\boldsymbol{h}-\boldsymbol{\mu}_{h}\right)\left(\boldsymbol{h}-\boldsymbol{\mu}_{h}\right)^{H}\right]$ is the channel covariance matrix. Throughout the paper we assume $\frac{2}{\sigma_{w}^{2}}$ is set to one to simplify the notation.

\section{ECRB for M OFDM Symbols}

Using a simple extension to our model in (1) for $M$ OFDM symbols, we obtain

$$
\left[\begin{array}{c}
\boldsymbol{Y}_{1} \\
\vdots \\
\boldsymbol{Y}_{M}
\end{array}\right]=\left[\begin{array}{ccc}
\boldsymbol{\Omega}_{1}\left(\tau_{d}\right) & \ldots & \mathbf{0} \\
\vdots & \ddots & \vdots \\
\mathbf{0} & \ldots & \boldsymbol{\Omega}_{M}\left(\tau_{d}\right)
\end{array}\right]\left[\begin{array}{c}
\boldsymbol{h}_{1} \\
\vdots \\
\boldsymbol{h}_{M}
\end{array}\right]+\left[\begin{array}{c}
\boldsymbol{W}_{1} \\
\vdots \\
\boldsymbol{W}_{M}
\end{array}\right]
$$

where

$$
\boldsymbol{\Omega}_{i}\left(\tau_{d}\right)=\boldsymbol{X}_{i} \boldsymbol{\Gamma}\left(\tau_{d}\right) \boldsymbol{F}_{L},
$$

and $\boldsymbol{X}_{i}$ is an $N \times N$ diagonal matrix of input at the the $i$ th OFDM symbol with the same definition as in (2), $\boldsymbol{h}_{i}$ is the channel coefficient vector of the $i$ th OFDM symbol assuming to be correlated with the channel coefficient vector of the $j$ th OFDM symbol $\boldsymbol{h}_{j}$, and $\boldsymbol{W}_{i}$ is a zero-mean complex Gaussian noise of the $i$ th OFDM symbol distributed as $\mathcal{C N}\left(\mathbf{0}, \sigma_{w}^{2} \boldsymbol{I}\right)$, and assumed to be independent of $\boldsymbol{W}_{j}$ for different values of $i$ and $j$. Applying the same approximation and assumption for the case of single OFDM symbol, we obtain the ECRB for $M$ OFDM symbols as

$$
\left[\begin{array}{c}
\widetilde{\operatorname{ECRB}}_{\tau_{d}} \\
\operatorname{tr}\left\{\widetilde{\operatorname{ECRB}}_{h}\right\}
\end{array}\right] \approx \frac{\sigma_{w}^{2}}{2}\left[\begin{array}{c}
\widetilde{J}_{\tau_{d}}^{-1} \\
\widetilde{J}_{h}
\end{array}\right],
$$

where

$$
\widetilde{J}_{\tau_{d}}=\operatorname{tr}\left\{\left(\widetilde{\boldsymbol{M}}_{1}-\widetilde{\boldsymbol{M}}_{2}\right) \widetilde{\boldsymbol{\Sigma}}_{h}\right\}+\widetilde{\boldsymbol{\mu}}_{h}^{H}\left(\widetilde{\boldsymbol{M}}_{1}-\widetilde{\boldsymbol{M}}_{2}\right) \widetilde{\boldsymbol{\mu}}_{h},
$$

and

$$
\widetilde{J}_{h}=2 \operatorname{tr}\left\{\widetilde{\boldsymbol{Q}}^{-1}\right\}+\widetilde{J}_{\tau_{d}}^{-1}\left(\operatorname{tr}\left\{\widetilde{\boldsymbol{M}}_{q} \widetilde{\boldsymbol{\Sigma}}_{h}\right\}+\widetilde{\boldsymbol{\mu}}_{h}^{H} \widetilde{\boldsymbol{M}}_{q} \widetilde{\boldsymbol{\mu}}_{h}\right),
$$

with $\widetilde{\boldsymbol{M}}_{1}=\operatorname{diag}\left\{\boldsymbol{M}_{1}^{(i)}\right\}_{i=1}^{M}, \quad \widetilde{\boldsymbol{M}}_{2}=\operatorname{diag}\left\{\boldsymbol{M}_{2}^{(i)}\right\}_{i=1}^{M}$, $\widetilde{\boldsymbol{M}}_{q}=\operatorname{diag}\left\{\boldsymbol{M}_{q}^{(i)}\right\}_{i=1}^{M}, \quad \widetilde{\boldsymbol{\mu}}_{h}=\left[\boldsymbol{\mu}_{1}^{T} \ldots \boldsymbol{\mu}_{M}^{T}\right]^{T}$ where $\boldsymbol{\mu}_{i}$ is the channel mean at $i$ th OFDM symbol, and $\widetilde{\boldsymbol{Q}}=$ $\operatorname{diag}\left\{\boldsymbol{Q}_{i}\right\}_{i=1}^{M}$. Also, we define $\boldsymbol{M}_{1}^{(i)}=\boldsymbol{F}_{L}^{H} \boldsymbol{D} \boldsymbol{P}_{i} \boldsymbol{D} \boldsymbol{F}_{L}$, $\boldsymbol{M}_{2}^{(i)}=\boldsymbol{F}_{L}^{H} \boldsymbol{P}_{i} \boldsymbol{D} \boldsymbol{F}_{L} \boldsymbol{Q}_{i}^{-1} \boldsymbol{F}_{L}^{H} \boldsymbol{P}_{i} \boldsymbol{D} \boldsymbol{F}_{L}, \boldsymbol{Q}_{i}=\boldsymbol{F}_{L}^{H} \boldsymbol{P}_{i} \boldsymbol{F}_{L}$, and $\boldsymbol{M}_{q}^{(i)}=\boldsymbol{F}_{L}^{H} \boldsymbol{P}_{i} \boldsymbol{D} \boldsymbol{F}_{L} \boldsymbol{Q}_{i}^{-1} \boldsymbol{Q}_{i}^{-1} \boldsymbol{F}_{L}^{H} \boldsymbol{P}_{i} \boldsymbol{D} \boldsymbol{F}_{L}$ where $\boldsymbol{P}_{i}=$ $\boldsymbol{X}_{i}^{H} \boldsymbol{X}_{i}$ is an $N \times N$ diagonal matrix representing pilot powers of the $i$ th OFDM symbol. Finally, $\widetilde{\boldsymbol{\Sigma}}_{h}$ represents a BlockToeplitz matrix [10] defined as

$$
\widetilde{\boldsymbol{\Sigma}}_{h}=\left[\begin{array}{cccc}
\boldsymbol{\Sigma}_{0} & \boldsymbol{\Sigma}_{1} & \ldots & \boldsymbol{\Sigma}_{M-1} \\
\boldsymbol{\Sigma}_{1}^{H} & \ddots & \ddots & \boldsymbol{\Sigma}_{M-2} \\
\vdots & \ddots & \ddots & \vdots \\
\boldsymbol{\Sigma}_{M-1}^{H} & \boldsymbol{\Sigma}_{M-2}^{H} & \ldots & \boldsymbol{\Sigma}_{0}
\end{array}\right]
$$

with $\left(\boldsymbol{\Sigma}_{k}\right)_{k=0, \ldots, M-1}$ are $L \times L$ matrices (not necessarily Toeplitz) representing channel covariance matrix of symbol $i$ for $k=0$ and cross correlation matrices between different OFDM symbols for $k \neq 0$. This is due to the fact that channel has the same covariance matrix for different OFDM symbols and cross correlation matrix between symbols $i$ and $j$ depends on $i-j$. However, channel coefficients can have different means $\boldsymbol{\mu}_{i=1, \ldots, M}$ for different OFDM symbols. The entries 
of each sub matrix $\boldsymbol{\Sigma}_{k}$ are of the form $\left(\boldsymbol{\Sigma}_{k}\right)_{u, v}=\sigma_{u, v}^{k}$, for $u, v=1, \ldots, L$. Consequently, (9) and (10) can be written as

$$
\begin{gathered}
\widetilde{J}_{\tau_{d}}=\sum_{i=1}^{M}\left[\operatorname{trace}\left\{\left(\boldsymbol{M}_{1}^{(i)}-\boldsymbol{M}_{2}^{(i)}\right) \boldsymbol{\Sigma}_{0}\right\}\right. \\
\left.+\boldsymbol{\mu}_{i}^{H}\left(\boldsymbol{M}_{1}^{(i)}-\boldsymbol{M}_{2}^{(i)}\right) \boldsymbol{\mu}_{i}\right] . \\
\widetilde{J}_{h}=\sum_{i=1}^{M}\left[2 \operatorname{tr}\left\{\boldsymbol{Q}_{i}^{-1}\right\}+\widetilde{J}_{\tau_{d}}^{-1}\left(\operatorname{tr}\left\{\boldsymbol{M}_{q}^{(i)} \boldsymbol{\Sigma}_{0}\right\}\right.\right. \\
\left.\left.+\boldsymbol{\mu}_{i}^{H} \boldsymbol{M}_{q}^{(i)} \boldsymbol{\mu}_{i}\right)\right] .
\end{gathered}
$$

From (12) and (13) it turns out that channel correlation $\boldsymbol{\Sigma}_{k \neq 0}$ does not affect the ECRB. Next, we propose an alternative approach to consider the effect of channel variations by changing channel covariance and mean for a given channel power and design the pilots based on the new model.

\section{Pilot Design}

In this section, first a model for channel uncertainty between different OFDM symbols for a given channel power is presented. Second, we propose a pilot design based on signal to interference and noise ratio (SINR) (the effect of channel estimation error is considered as interference) for a given accuracy in the estimation of time-delay. Note that we assume time-delay $\tau_{d}$ is fixed within estimation phase and channel variations is due to fast variations of the amplitudes [11] that changes channel statistics, i.e. channel mean and covariance for different OFDM symbols.

\section{A. Modeling the Channel Uncertainty}

We propose a novel method to model channel uncertainty between different OFDM symbols by scaling channel mean and covariance for a given power $P_{h}$ (this is due to the fact that channel power between different OFDM symbols does not change) defined as $\mathbb{E}\left[\|\boldsymbol{h}\|^{2}\right]$, the operation $\|$.$\| is the norm of$ a vector. A coefficient $a^{2}$ is defined to change the channel covariance as $a^{2} \boldsymbol{\Sigma}_{h}$, and a coefficient $b$ to change the channel mean as $b \boldsymbol{\mu}_{h}$. Using the expected value of a quadratic function, the total channel power $P_{h}$ is

$$
P_{h}=a^{2} \epsilon^{2}+b^{2} \mu^{2},
$$

where

$$
\begin{gathered}
\epsilon^{2}=\operatorname{tr}\left\{\boldsymbol{\Sigma}_{h}\right\}=\mathbb{E}\left[\left\|\boldsymbol{h}-\boldsymbol{\mu}_{h}\right\|^{2}\right], \\
\mu^{2}=\boldsymbol{\mu}_{h}^{H} \boldsymbol{\mu}_{h} .
\end{gathered}
$$

Actually, (14) means that we are scaling the covariance with the coefficient $a^{2}$ and the mean squared with the coefficient $b^{2}$ in such a way that their sum remains constant. From (14), for a fixed value of $b^{2}$ the coefficient $a^{2}$ can be written as

$$
a^{2}=\frac{P_{h}-b^{2} \mu^{2}}{\epsilon^{2}}
$$

The coefficients $a^{2}$ and $b^{2}$ are used in the next part to investigate the effects of channel uncertainty on pilot design.

\section{B. Optimization Problem for Pilot Design}

The design problem can be formulated as

$$
(P 1)\left\{\begin{array}{cc}
\max _{\boldsymbol{p}_{p}, \boldsymbol{p}_{d}} & \frac{1}{N} \sum_{k \in \mathbb{D}} \operatorname{SINR}_{k}\left(\boldsymbol{p}_{p}, \boldsymbol{p}_{d}, b\right) \\
\text { s.t. } & J_{\tau_{d}}^{-1}\left(\boldsymbol{p}_{p}, b\right) \leq \epsilon \\
& \mathbf{1}^{T} \boldsymbol{p}_{p}+\mathbf{1}^{T} \boldsymbol{p}_{d} \leq P_{t} \\
& \boldsymbol{p}_{p}^{T} \boldsymbol{p}_{d}=0 \\
& \boldsymbol{p}_{p} \succeq \mathbf{0} ; \boldsymbol{p}_{d} \succeq \mathbf{0},
\end{array}\right.
$$

where $\mathbf{1}$ is the all-one vector, $P_{t}$ is the total power, $\mathbb{D}$ is the set of subcarriers used for data transmission that is not fixed, $\boldsymbol{p}_{p}$ represents power allocated to the subcarriers for estimation or pilots, $\boldsymbol{p}_{d}$ stands for power allocated to subcarriers for data transmission or data, and $\operatorname{SINR}_{k}\left(\boldsymbol{p}_{p}, \boldsymbol{p}_{d}, b\right)$ is SINR for $k$ th subcarrier of a given channel variability parameter $b$ defined as

$$
\operatorname{SINR}_{k}\left(\boldsymbol{p}_{p}, \boldsymbol{p}_{d}, b\right)=\frac{p_{d, k}|H(k)|^{2}}{p_{d, k} \boldsymbol{F}_{k, L} \boldsymbol{J}_{h}\left(\boldsymbol{p}_{p}, b\right) \boldsymbol{F}_{k, L}^{H}+\sigma^{2}},
$$

where $p_{d, k}$ is the $k$ th entry of data vector $\boldsymbol{p}_{d}, \boldsymbol{F}_{k, L}$ is the $k$ th row of $\boldsymbol{F}_{L}$ representing first $L$ columns of discrete Fourier transform (DFT) matrix (Note that the term $F_{k, L} \boldsymbol{J}_{h}\left(\boldsymbol{p}_{p}, b\right) \boldsymbol{F}_{k, L}^{H}$ is interpolation of the estimated values of channel coefficients to obtain the value at the $k$ th subcarrier for data transmission), and $H(k)=\boldsymbol{F}_{k, L} \boldsymbol{h}$. Also, $J_{\tau_{d}}\left(\boldsymbol{p}_{p}, b\right)$ and $J_{h}\left(\boldsymbol{p}_{p}, b\right)$ are defined as

$$
\begin{aligned}
J_{\tau_{d}}\left(\boldsymbol{p}_{p}, b\right)=a^{2} \operatorname{tr}\{ & \left.\left(\boldsymbol{M}_{1}-\boldsymbol{M}_{2}\right) \boldsymbol{\Sigma}_{h}\right\} \\
& +b^{2} \boldsymbol{\mu}_{h}^{H}\left(\boldsymbol{M}_{1}-\boldsymbol{M}_{2}\right) \boldsymbol{\mu}_{h},
\end{aligned}
$$

and

$$
\begin{array}{r}
\boldsymbol{J}_{h}\left(\boldsymbol{p}_{p}, b\right)=2 \boldsymbol{Q}^{-1}+J_{\tau_{d}}^{-1}\left(\boldsymbol{p}_{p}, b\right)\left(a^{2} \boldsymbol{M}_{q} \boldsymbol{\Sigma}_{h}\right. \\
\left.+b^{2} \boldsymbol{M}_{q} \boldsymbol{\mu}_{h} \boldsymbol{\mu}_{h}^{H}\right)
\end{array}
$$

where $a^{2}$ is defined in (17). The first constraint in problem (P1) limits the minimum accuracy in the estimation of timedelay to $\epsilon$, second constraint force the total power for data and pilot to be less than or equal to $P_{t}$, the third constraint makes the problem a combinatorial optimization that is a nonconvex problem, and the last constraint is due to positive values of data and pilot. Instead of solving the non-convex optimization problem $(\mathrm{P} 1)$, we solve the following relaxed optimization problem obtained by omitting the third constraint in (P1). By omitting the third constraint in (P1) and solving the relaxed problem (P2) only a few subcarriers are used as pilots with higher amplitudes and the rest of subcarriers with smaller amplitudes are set for data transmission.

$$
(P 2)\left\{\begin{array}{cc}
\max _{\boldsymbol{p}_{p}, \boldsymbol{p}_{d}} & \frac{1}{N} \sum_{k \in \mathbb{D}} \operatorname{SINR}_{k}\left(\boldsymbol{p}_{p}, \boldsymbol{p}_{d}, b\right) \\
\text { s.t. } & J_{\tau_{d}}^{-1}\left(\boldsymbol{p}_{p}, b\right) \leq \epsilon \\
& \mathbf{1}^{T} \boldsymbol{p}_{p}+\mathbf{1}^{T} \boldsymbol{p}_{d} \leq P_{t} \\
& \boldsymbol{p}_{p} \succeq \mathbf{0} ; \boldsymbol{p}_{d} \succeq \mathbf{0} .
\end{array}\right.
$$


Problem (P2) can be written in a semi-definite programming (SDP) form using the Schur complement formula as

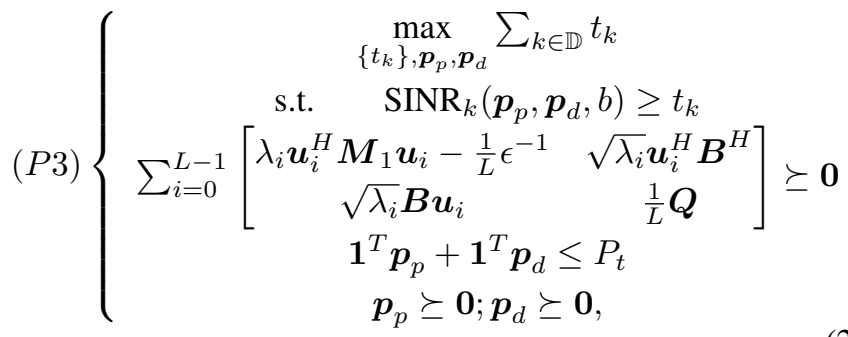

with $\lambda_{i}$ and $\boldsymbol{u}_{i}$ being the $i$ th eigenvalue and eigenvector of $a^{2} \boldsymbol{\Sigma}_{h}+b^{2} \boldsymbol{\mu}_{h} \boldsymbol{\mu}_{h}^{H}$ respectively, $t_{k}$ is an auxiliary parameter, and $\boldsymbol{B}$ is defined as $\boldsymbol{F}_{L}^{H} \boldsymbol{P} \boldsymbol{D} \boldsymbol{F}_{L}$. Problem (P3) is a convex optimization problem due to the linear cost function and the fact that the constraints are in the form convex functions. Note that at sufficiently high $\operatorname{SNR}\left(P_{d, k} / \sigma^{2} \gg 1\right.$ for the constraint to be active or $P_{d, k}>0$ ) the first constraint can be written as $t_{k} \boldsymbol{F}_{k, L} \boldsymbol{J}_{h}\left(\boldsymbol{p}_{p}, b\right) \boldsymbol{F}_{k, L}^{H} \leq|H(k)|^{2}$ that is a convex function with respect to $\boldsymbol{p}_{p}$, i.e. it is of the form of a matrix fractional function that is convex [12].

It can be proved that by increasing factor $b$ (increasing channel mean $\boldsymbol{\mu}_{h}$ and reducing channel covariance $\boldsymbol{\Sigma}_{h}$ for a given channel power $\left.P_{h}\right)$ both $J_{\tau_{d}}^{-1}\left(\boldsymbol{p}_{p}, b\right)$ and $J_{h}\left(\boldsymbol{p}_{p}, b\right)$ decrease, see the Appendix for comparing the results for $b^{2}=0$ and $b^{2}=P_{h} / \mu^{2}$. This means the constraint $J_{\tau_{d}}^{-1}\left(\boldsymbol{p}_{p}, b\right) \leq \epsilon$ is fulfilled with less amount of the total power $P_{t}$ for the same time-delay accuracy. Also, decreasing $J_{h}\left(\boldsymbol{p}_{p}, b\right)$ by increasing $b$ leads to higher value of $\operatorname{SINR}_{k}\left(\boldsymbol{p}_{p}, \boldsymbol{p}_{d}, b\right)$ for a given total power $P_{t}$ based on the formulation in (19). Consequently, one can obtain the same time-delay accuracy and per-symbol SINR using less amount of total power $P_{t}$.

\section{Simulation Results}

In this section, the effect of different channel mean $\boldsymbol{\mu}_{h}$ and covariance $\boldsymbol{\Sigma}_{h}$ on the bounds, capacity, and total power is investigated. The channel covariance matrix is $\boldsymbol{\Sigma}_{h}=\frac{1}{L} \boldsymbol{I}$. We assume a channel impulse response of length $L=4$ with the mean value of $\boldsymbol{\mu}_{h}^{T}=[1,0,0,0]$ and an OFDM signal with $N=40$ subcarriers. The total power for joint data and pilot design is set to $P_{t}=5$.

Note that only to reduce the simulations time for joint data and pilot design we use $P_{t}=5$ and minimum estimation accuracy of time-delay of the order $10^{-3}$. Obviously, one can increase the total power and apply minimum estimation accuracy of time-delay of the order $10^{-9}$ or smaller values that are closer to realistic values of time-delay used for positioning purposes. Figure 1 studies the effect of different values of $b$ on $J_{\tau_{d}}^{-1}$ and $J_{h}$. Note that the values of $J_{\tau_{d}}^{-1}$ and $J_{h}$ are normalized by multiplying the results with $\left(\frac{N}{T_{s}}\right)^{2}$ and $\frac{1}{L}$ respectively. It can be observed that by increasing the value of $b$ or equivalently by reducing channel covariance $\Sigma_{h}$ and improving the channel mean $\boldsymbol{\mu}_{h}$ for a fixed power $P_{h}$, both $J_{\tau_{d}}^{-1}$ and $J_{h}$ decrease.

Figure 2 shows the percent of required increase in the total power $P_{t}$ for $b^{2}=0$ to obtain the same time-delay estimation
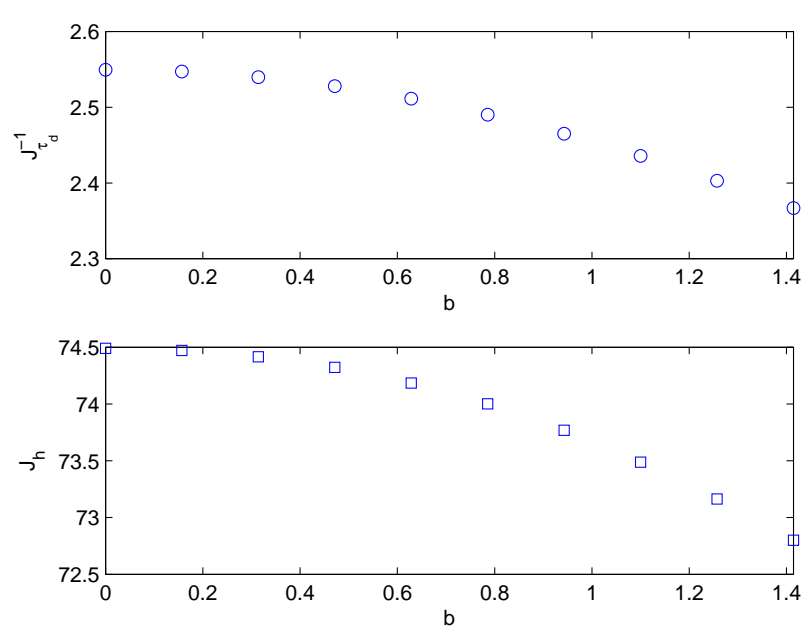

Fig. 1. Decreasing patterns of (top) $J_{\tau_{d}}^{-1}$ and (bottom) $J_{h}$ by increasing $b$.

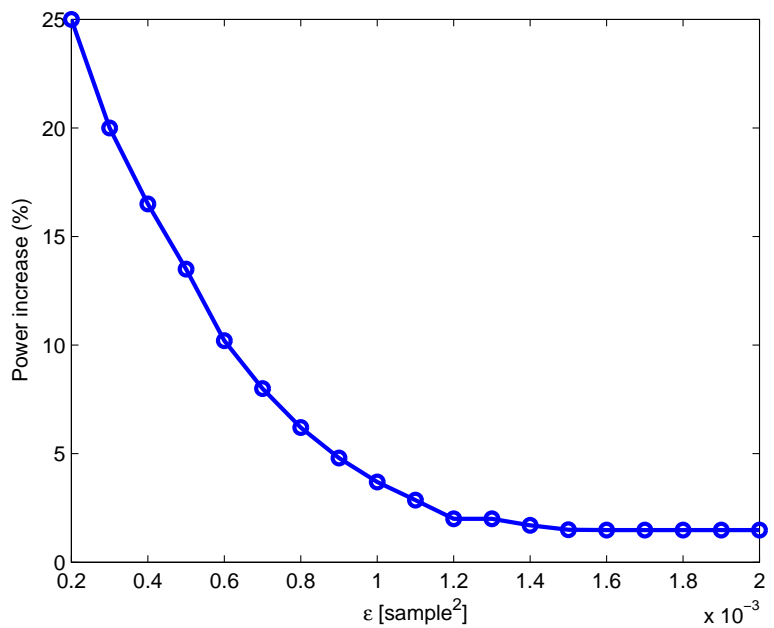

Fig. 2. Total power increase required for $b^{2}=0$ to obtain the same timedelay accuracy and per-symbol SINR as $b^{2}=P_{h} / \mu^{2}$.

accuracy and per-symbol SINR as in $b^{2}=P_{h} / \mu^{2}$. In other words, to achieve the same data transmission rate and timedelay estimation accuracy for the case of $b^{2}=P_{h} / \mu^{2}$, one needs to increase the total power $P_{t}$ for the case of $b^{2}=0$. Intuitively, this means that the more we are uncertain about the channel, the more we need to increase total power $P_{t}$ to obtain the same performance. Also, the amount of required increase in the total power $P_{t}$ increases by more time-delay estimation accuracy $\epsilon$, i.e. smaller values of $\epsilon$.

Finally, Figure 3 shows the increasing pattern of the lower bound of capacity which is defined as a logarithmic function of per-symbol SINR as

$$
C_{l b}=\frac{1}{N} \sum_{k \in \mathbb{D}} \log \left(1+\operatorname{SINR}_{k}\left(\boldsymbol{p}_{p}, \boldsymbol{p}_{d}, b\right)\right),
$$

with respect to upper bound of time-delay estimation accuracy $\epsilon$ for $b^{2}=0$ and $b^{2}=P_{h} / \mu^{2}$. It is clear that the capacity is 

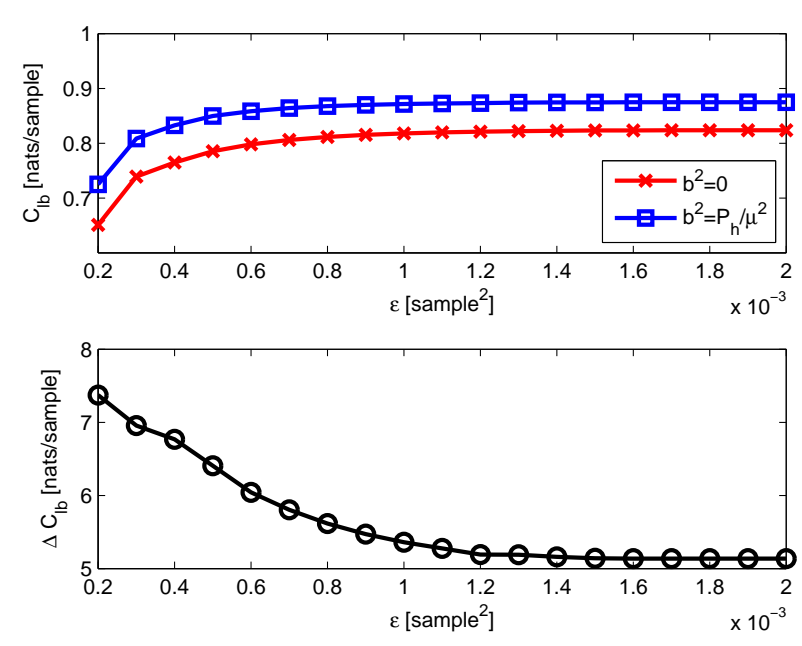

Fig. 3. (top) Increasing pattern of the lower bound of capacity $C_{l b}$ with respect to minimum time-delay estimation accuracy $\epsilon$ for $b^{2}=0$ and $b^{2}=$ $P_{h} / \mu^{2}$, and (bottom) the difference between lower bound of capacity for $b^{2}=0$ and $b^{2}=P_{h} / \mu^{2}\left(\Delta C_{l b}\right)$ with respect to minimum time-delay estimation accuracy $\epsilon$.

increased for all the values of time-delay estimation accuracy for $b^{2}=P_{h} / \mu^{2}$. Also, the increase in the amount of capacity is more evident for higher time-delay estimation accuracy, i.e. smaller values of $\epsilon$. The bottom plot shows the percentage of difference between lower bound of capacity for $b^{2}=0$ and $b^{2}=P_{h} / \mu^{2}$.

\section{CONCLUSION}

A model for channel variability of different OFDM symbols is proposed based on varying channel mean and covariance for a given channel power. The effect of channel variability on the joint design of pilot and data power allocations is investigated. Joint design of data and pilot power allocations is turned from a combinatorial (non-convex) optimization to an SDP optimization (convex) problem. The results show that by reducing channel covariance and increasing channel mean for a given channel power the estimation accuracy of channel and time-delay is improved. Also, in terms of saving the total power for a given time-delay accuracy and per-symbol SINR one can save up to $25 \%$ for the case of knowing the exact value of channel mean with channel covariance matrix of zero. Finally, simulations verify increasing the capacity by increasing channel mean and reducing channel covariance. Consequently, in an OFDM system with correlated OFDM symbols one can use the information from the previous symbols to improve the estimation accuracy and per-symbol SINR.

\section{APPENDIX}

Considering the fact that estimated channel coefficients $\hat{\boldsymbol{h}}$ and error in the estimation $e$ are related to the actual channel coefficients $\boldsymbol{h}$ as $\boldsymbol{h}=\hat{\boldsymbol{h}}+\boldsymbol{e}$, and also noting that error in the estimation of channel coefficients $e$ is a zero-mean Gaussian variable with $\mathbb{E}\left[\boldsymbol{e} \boldsymbol{e}^{H}\right]=\sigma_{e}^{2} \boldsymbol{I}$, one can obtain

$$
\boldsymbol{\Sigma}_{h}=\mathbb{E}\left[\boldsymbol{e} \boldsymbol{e}^{H}\right]=\sum_{i=0}^{L-1} \sigma_{e}^{2} \boldsymbol{v}_{i} \boldsymbol{v}_{i}^{H}
$$

and

$$
\boldsymbol{\mu}_{h} \boldsymbol{\mu}_{h}^{H}=\|\boldsymbol{h}\|^{2} \boldsymbol{u} \boldsymbol{u}^{H},
$$

where $\boldsymbol{v}_{i}$ represents the $i$ th eigenvector defined as an all-zero vector of length $L$ with $i$ th entry of one, $\|\boldsymbol{h}\|^{2}$ is the channel norm, and $\boldsymbol{u}$ is th6e eigenvector with $\boldsymbol{u}^{H} \boldsymbol{u}=1$. So, in order to prove the value of $J_{\tau_{d}}^{-1}$ and $J_{h}$ for $b^{2}=P_{h} / \mu^{2}$ is smaller than the corresponding values for $b^{2}=0$, it is sufficient to show that $\boldsymbol{M}\left(\|\boldsymbol{h}\|^{2} \boldsymbol{u} \boldsymbol{u}^{H}-\sigma_{e}^{2} \boldsymbol{I}\right) \succeq \mathbf{0}$ (Multiplication of two hermitian and positive semi-definite matrices (PSD) is a PSD matrix) where $M$ stands for a hermitian and PSD matrix that can be replaced by $M_{1}-M_{2}$ and $M_{q}$ for $J_{\tau_{d}}$ and $J_{h}$ respectively. This is always true using the definition of a PSD matrix provided that $\left.\|\boldsymbol{h}\|\right|^{2}\|\boldsymbol{z}\|^{2}-\sigma_{e}^{2}\|\boldsymbol{x}\|^{2} \geq 0$ for $\boldsymbol{x}$ being an arbitrary vector and $\|\boldsymbol{z}\|^{2}=\boldsymbol{x}^{H} \boldsymbol{u} \boldsymbol{u}^{H} \boldsymbol{x}$. This condition is always fulfilled practically since $\sigma_{e}^{2}$ is sufficiently small.

\section{REFERENCES}

[1] R. Negi and J. Cioffi, "Pilot tone selection for channel estimation in a mobile OFDM system," IEEE Transactions on Consumer Electronics, vol. 44, pp. 1122-1128, Aug. 1998.

[2] I. Barhumi, G. Leus, and M. Moonen, "Optimal training design for MIMO OFDM systems in mobile wireless channels," IEEE Transactions on Signal Processing, vol. 51, no. 6, pp. 1615-1624, Jun. 2003.

[3] H. Minn and N. Al-Dhahir, "Optimal training signals for MIMO-OFDM channel estimation," IEEE Transactions on Wireless Communications, vol. 5, no. 5, pp. 1158-1168, May. 2006.

[4] H. Minn and S. Xing, "An optimal training signal structure for frequency-offset estimation," IEEE Transactions on Communications, vol. 53, no. 2, pp. 343-355, Feb. 2005.

[5] P. Stoica and O. Besson, "Training sequence design for frequency offset and frequency-selective channel estimation," IEEE Transactions on Communications, vol. 51, no. 11, pp. 1910-1917, Nov. 2003.

[6] A. A. Nasir, H. Mehrpouyan, S. Durrani, S.D. Blostein, R.A. Kennedy, and B. Ottersten, "Optimal training sequences for joint timing synchronization and channel estimation in distributed communication networks," IEEE Transactions on Communications, vol. 61, no. 7, pp. 3002-3015, Jul. 2013.

[7] M.D. Larsen, G. Seco-Granados, and A.L. Swindlehurst, "Pilot optimization for time-delay and channel estimation in OFDM systems," in IEEE International Conference on Acoustics, Speech and Signal Processing (ICASSP). 2011, pp. 3564-3567, IEEE.

[8] R. Montalban, J. A. Lopez-Salcedo, G. Seco-Granados, and A. L. Swindlehurst, "Power allocation method based on the channel statistics for combined positioning and communications OFDM systems," in IEEE International Conference on Acoustics, Speech and Signal Processing (ICASSP). 2013, pp. 4384-4388, IEEE.

[9] D. T. Pham, J. Verron, and M. C. Roubaud, "A singular evolutive extended kalman filter for data assimilation in oceanography," Elsevier Journal of Marine Systems, vol. 16, no. 3-4, pp. 323-340, Oct. 1998.

[10] H. Gazzah, P. A. Regalia, and J. P. Delmas, "Asymptotic eigenvalue distribution of block toeplitz matrices and application to blind SIMO channel identification," IEEE Transactions on Information Theory, vol. 47, no. 3, pp. 1243-1251, Mar. 2001.

[11] O. Simeone, Y. Bar-Ness, and U. Spagnolini, "Pilot-based channel estimation for OFDM systems by tracking the delay-subspace," IEEE Transactions on Wireless Communications, vol. 3, no. 1, pp. 315-325, Jan. 2004.

[12] S. Boyd and L. Vandenberghe, Convex Optimization, Cambridge University Press, New York, NY, USA, 2004. 